\title{
Erratum to: An update on peripheral ossifying fibroma: case report and literature review
}

\author{
María José Franco-Barrera ${ }^{1}$ - María Guadalupe Zavala-Cerna ${ }^{2}$. \\ Rubén Fernández-Tamayo ${ }^{1}$ - Israel Vivanco-Pérez ${ }^{1}$. \\ Nora Mariana Fernández-Tamayo ${ }^{3,4}$. Olivia Torres-Bugarín ${ }^{5}$
}

Published online: 8 November 2016

(C) Springer-Verlag Berlin Heidelberg 2016

Erratum to: Oral and Maxillofacial Surgery 20(1): 1-7

DOI: 10.1007/s10006-015-0535-0

The original version of the above article contained a mistake in reference 1. Correct reference is presented here:

The online version of the original article can be found at http://dx.doi. org/10.1007/s10006-015-0535-0.

\footnotetext{
María Guadalupe Zavala-Cerna

g_zavala_78@hotmail.com

$\triangle$ Olivia Torres-Bugarín

oliviatorres@hotmail.com

María José Franco-Barrera

mafraba88@hotmail.com

Rubén Fernández-Tamayo

ruferwolf@gmail.com

Israel Vivanco-Pérez

vivancoperezcm@gmail.com

Nora Mariana Fernández-Tamayo

dredgarsantos@hotmail.com

1 Maxilofacial Surgery Division, Hospital Universitario de Puebla, Calle 13 Sur 1301, 72410 Heróica Puebla de Zaragoza Pue, Mexico

2 Immunology Department, School of Medicine, Universidad Autonoma de Guadalajara, Av. Patria 1201, Lomas del Valle, 45129 Zapopan, Jalisco, Mexico

3 Pathology Laboratory, Benemerita Universidad Autonoma de Puebla, Puebla, Mexico

4 Pathology Laboratory, Hospital ISSSTEP, Av Emiliano Zapata, San Baltazar Campeche, 72550 Heróica Puebla de Zaragoza Pue, Mexico

5 Genotoxic Research Laboratory at School of Medicine, Universidad Autonoma de Guadalajara, Av. Patria 1201, Lomas del Valle, 45129 Zapopan, Jalisco, Mexico
}

Barnes L, Eveson JW, Reichart P, Sidransky D. World Health Organization classification of tumors: pathology and genetics, head and neck tumors. Lyon: IARC Press; 2005 . 